\title{
The Role of Computed Tomography \& Magnetic Resonance Imaging and Ultrasongraphy in detection of Gastrointestinal tract tumors
}

\author{
Maha Esmeal Ahmed Esmeal \\ Ph. D, Assistant Professor -Radiological Sciences Department-Applied Medical College,Najran University- \\ Kingdom of Saudi Arabia
}

\begin{abstract}
:
Computed tomography $(C T)$ scanning is typically used in the evaluation of patients with known colonic neoplasm's or in the detection of colorectal tumors in patients with nonspecific abdominal complaints. Magnetic resonance imaging (MRI) is extremely helpful in detection and characterization of suspected hepatic metastases, particularly in the setting of a negative CT scan. MRI is also effective in the staging of tumor for local extension. Transrectal ultrasonography has become popular in staging tumors for local extension because of its ability to demonstrate the various layers of the colon wall. The incidence of gasterointestinal tract tumors is expected to increase in the future.

This research paper is focus on the role of Computed tomography (CT scan),Magnetic resonance Imaging (MRI) and Ultrasound (u/s) in detection of tumors in the human body.The evaluation of of (CT scan), (MRI) and Ultrasound can provide valuable information regarding GIT tumors . So the final diagnosis depend and based on imaging modalities for detection GIT tumors. The various imaging modalities that used for detection of GIT tumors. Researcher also discuss the various imaging characteristics after administration of contrast agent .MRI like CT scan has difficulty in diagnosing metastases local lymph node involvement however, transrectal ultrasonography (TRUS) has recently became popular in staging colonic malignancy.

Key-words: Role Computed tomography Ultrasound tumors Magnetic resonance Imaging
\end{abstract}

\section{Computed Tomography:}

\section{Introduction :}

Computed tomography scanning has become the workhorse diagnostic imaging modality in detecting, staging, and following patients with cancer of the gastrointestinal tract tumors(Figure 1-1).. It is an efficient means of obtaining a global assessment of the entire abdomen and pelvis. In addition, CT scanning can determine the thickness of the colonic wall involved with tumor and depict the relationship of the neoplasm with surrounding structures. Metastases to adjacent structures, adrenal glands, lymph nodes, liver, bony structures, and musculature can be ascertained. Studies consistently indicate that CT is an excellent means of initially staging primary or secondary colorectal tumor. The sensitivity of detecting hepatic metastases has been well documented in the literature, with rates ranging from 85 to 90 percent.(1)Accuracy rates have been reported around 70 percent for determining local tumor extension.(2) CT scanning is particularly useful in staging patients with Dukes stage D lesions, which may lead to changes in surgical planning or preoperative management. Positive predictive value rates have been reported at 100 percent for CT staging of Dukes D lesions.(3) Frequently, however, CT may under stage patients with microinvasion of pericolonic or perirectal fat or small tumor foci in normal-sized nodes. Lymph node sensitivity detection has been reported as low as 25.9 percent.(4)

Advances in CT technology have enabled scanners to obtain images in less than 1 second per slice. Helical CT scanning produces continuous three dimensional CT information by allowing the table to move continuously through a rotating radiograph producing gantry. Information can then be formatted in variable slice thickenesses since a helix, or volume of information, is obtained. Shorter scan times, reduction in patient organ motion, and the ability to acquire images rapidly after intravenous contrast enhancement are advantages of the newer generation CT scanners.(5)

A variety of imaging techniques can be used to enhance images obtained by $\mathrm{CT}$. intravenous contrast is extremely important in achieving differences in conspicuity between normal and abnormal tissue. Two types of intravenous contrast media are typically available: ionic and nonionic. Nonionic contrast is generally preferred because of its lower incidence of adverse allergic reactions. Adequate opacification of the alimentary tract is required for assessment of mucosal detail in the colon. Adequate distention of the colon can usually be achieved when the patient receives approximately $700 \mathrm{cc}$ of contrast orally. The administration of rectal contrast has also proven helpful in detection and staging of colorectal neoplasm (5). 
Computed tomography scanning is a cost effective means of evaluating the entire abdomen and pelvis. The average cost of an abdominal and pelvic CT scan ranges from US $\$ 300$ to US $\$ 1,000$. The ease of performing and scheduling a CT has resulted in a 75 percent increase in the volume of examinations over the past 5 years at different institution. As previously mentioned, CT is inadequate in its ability to accurately detect microscopic foci of disease. Images from CT scans can be degraded by artifacts. For example, beam-hardening artifact from a hip prosthesis can markedly impair evaluation of the pelvis. CT scanning, however, continues to be the preferred imaging modality at different institution for staging colorectal neoplasm (5).

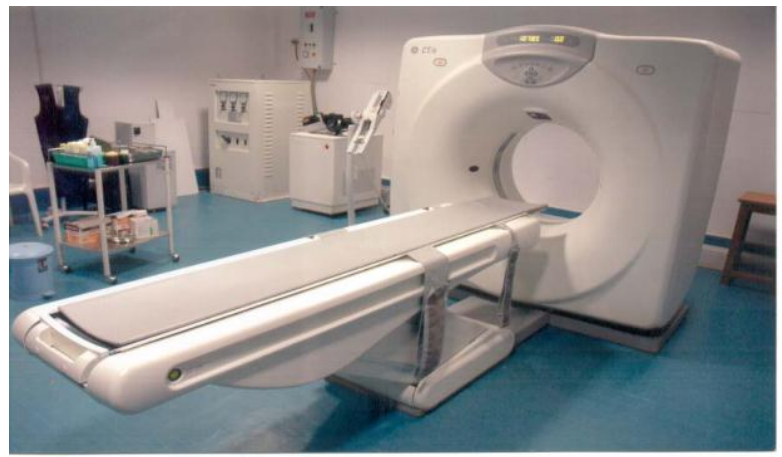

Figure 1-1. Images of the CT scan unit

\section{Magnetic Resonance Imaging:}

Magnetic resonance imaging is becoming increasingly popular in the diagnosis and staging of colorectal techniques, such as superior phased array pelvic and endorectal coils as well as the administration of rectal contrast agents, has improved image quality(Figure 1-2).. The multiplanar imaging capabilities of MRI offer a special advantage of this modality. Images produced by MRI are based on the interactions between protons in tissues within an alternating magnetic field. Hydrogen protons become excited when a specific radio frequency (RF) is applied. These protons subsequently return to equilibrium (relaxation), resulting in a release of RF energy called an echo. The relaxation of hydrogen protons to equilibrium has been designated T1 and T2. cancer. The advent of new imaging tech- MRI images can be varied by changing the interval between RF pulses (TR) and the time between the RF pulse and the signal reception (TE). T1-weighted images are formed by keeping the TR and TE shorter; T2-weighted images are produced by keeping TR and TE longer. Different biologic tissues have characteristic MRI appearances. Free water, such as seen in edema, have a long T1 and T2 relaxation and will appear low in signal intensity on T1-weighted images and high in signal intensity on T2weighted images. Fat behaves differently and demonstrates short T1 relaxation times. Fat therefore appears high in signal intensity on T1-weighted images. Gadolinium is aparamagnetic contrast agent that causes a reduction in T1 and T2 relaxation times, resulting in a higher signal on T1-weighted images. Studies have shown MRI to be equally effectivein staging colorectal tumors when compared to CT.(6)

Overall staging accuracies have been reported at 74 to 82 percent.(6-9) Although only a few studies with small patient populations have been reported using endorectal coils for staging colorectal cancer, initial results have been encouraging, with sensitivities reported at 92 percent for the assessment of local tumor extension(10) (Figure 1-3). MRI has proven particularly useful in the search for hepatic metastases when CT results are technically unsatisfactory or when clinical evaluation is in conflict with good quality CT results.(11) Favorable results have been reported in staging patients with recurrent colorectal neoplasm, with accuracies reported in the 95 percent range.(12) MRI, like CT, has difficulty in diagnosing metastatic local lymph node involvement, with accuracies of correctly diagnosing lymph nodes reported around 60 percent.(13) Perirectal fat infiltration is also difficult to diagnosis with reported accuracies of only 71 percent for T3 tumors.(14) 


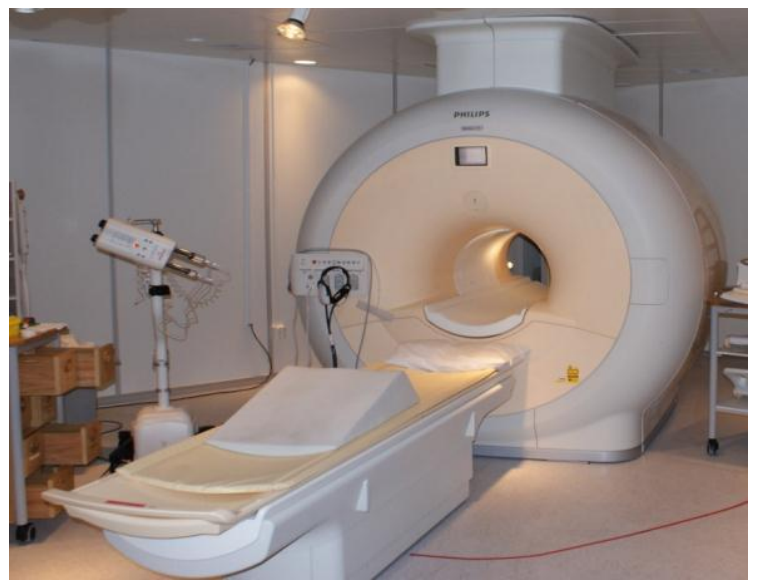

Figure 1-2. Images of the MRI unit

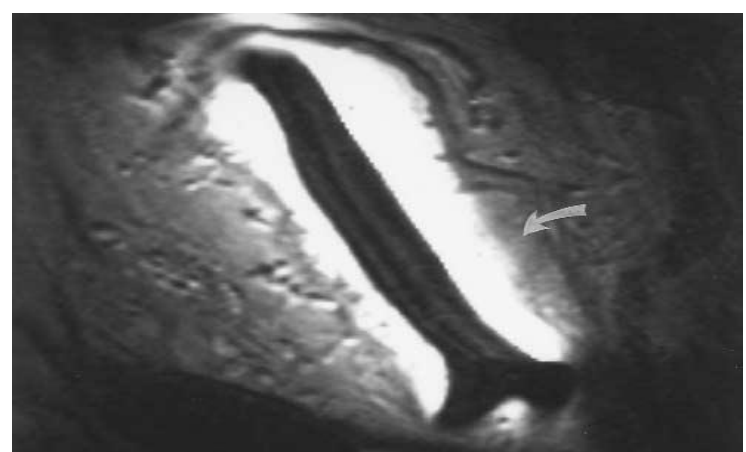

Figure 1-3. Images of the rectum obtained with an endorectal coil in place demonstrating marked thickening of the rectum representing

a large adenocarcinoma.

\section{Ultrasonography:}

Transrectal ultrasonography (TRUS) has recently become popular in staging colonic malignancy(Figure 1-4). TRU Sallows visualization of the layers of the colonic wall; therefore, the depth of tumor extension can be ascertained. Pericolonic nodes and extension beyond the serosa can often be diagnosed with TRUS. Sensitivities ranging from 67 to 96 percent for detection of tumor spread beyond the rectal wall have been reported. $(4,15,16)$ Detection of local adenopathy has been reported at 50 to 75 percent $(4,15,16)$ .Although perirectal infiltration of tumor is usually detected with sensitivities as high as 97 percent, the specificity of examining the perirectal region is low, with numbers reported at 24 percent. (17) Advanced neoplasms are often difficult to evaluate with TRUS and are generally better assessed with CT or MRI. Images produced from TRUS appear as rings radiating from a transducer that is placed within the colonic lumen. The transducer is covered with a balloon filled with water that appears hypoechoic (black).(5) The various layers of the colonic wall produce different levels of echogenicity. The innermost ring appears as a hyperechoic band (white) and represents the interface of the balloon with mucosa.(5)

The second layer seen appears relatively hypoechoic and represents the muscularis mucosa.(5) The third layer is hyperechoic and represents the submucosa. .(5) The fourth ring seen is hypoechoic and represents the muscularis propria. (5) The fifth layer is hyperechoic and represents the interface between pericolonic fat and the serosa of the colon. (5)

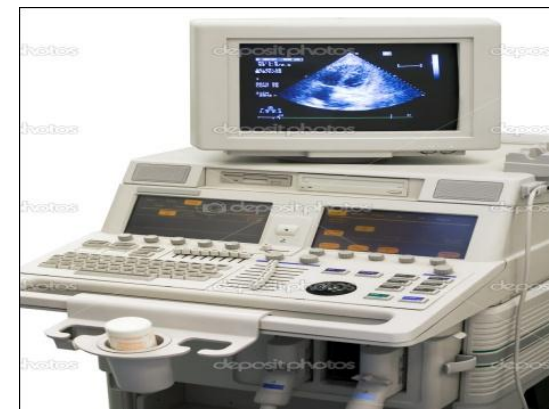

Figure 1-4. Images of the Ultrasound unit 


\section{Conclusion:}

A variety of radiologic imaging studies are available in the diagnosis of GIT neoplasm's. The selection of the appropriate study should be tailored to the individual patient. The institutional availability and expertise largely governs which modality should be used. Presently, no one imaging modality adequately detects and stages GIT neoplasm's. Studies should be considered complementary and institutional algorithms should be created for maximum patient benefit.

\section{Acknowledgement:}

Researchers would like to extend gratitude to the Dean ship and staff of the College of Medical Radiological Sciences, Najran University also would like to acknowledge all those who directly or indirectly extend support to this research. May almighty Allah make this research of special benefit to the development of health services all over the world

\section{References:}

[1]. Berland LL, Lewison TL, Foley WD, et al. Comparison of pre- and post-contrast CT in hepatic metastases. AJR Am J Roentgenol 1982;138:852-8

[2]. Thompson WM, Halvorsen RA, Foster WL, et al. Preoperative and postoperative CT staging of rectosigmoid carcinoma. AJR Am J Roentgenol 1986;146:703-10.

[3]. Bathazar EJ, Megibow AJ, Hulnick D, et al. Carcinomaof the colon: detection and preoperative staging by CT. AJR Am J Roentgenol 1988;150:301-6.

[4]. Freeney PC, Marks WM, Ryan JA, et al. Colorectal carcinoma evaluation with CT: preoperative staging and detection of postoperative recurrence. Radiology 1986;158:347-53.

[5]. Thoeni RF. Colorectal cancer radiologic staging. Radiol Clin North Am 1997;35:457-83.

[6]. Butch RJ, Stark DD, Wittenberg J, et al. Staging rectal cancer by MR and CT. AJR Am J Roentgenol 1986; 146:1155-60.

[7]. de Lange EE, Gechner RE, Edge SB, et al. Preoperativestaging of rectal carcinoma with MR imaging: surgical and histopathologic correlation. Radiology 1990; 176:623-8.

[8]. Guinet C, Buy JN, Ghossain MA, et al. Comparison of magnetic resonance imaging and computed tomography in the preoperative staging of rectal cancer. Arch Surg 1990;125:385-8.

[9]. Guinet C, Buy JN, Sezeur A, et al. Preoperative assessment of the extension of rectal carcinoma: correlation of the MR, surgical, and histopathologic findings. J Comput Assist Tomogr 1988;12:209-14.

[10]. Chan TW, Kressel HY, Milestone B, et al. Rectal carcinoma: staging at MR imaging with endorectal surface coil. Work in progress. Radiology 1991;181:461-7.

[11]. Demas BE, Hricak J, Goldberg HI, et al. Magnetic resonance imaging diagnosis of hepatic metastases in the presence of negative CT studies. J Clin Gastroenterol 1985;7:553-60.

[12]. Pema PJ, Bennett WF, Bova JG, et al. CT vs MRI in diagnosis of recurrent rectosigmoid carcinoma. J Comput Assist Tomogr 1994;18:256-61.

[13]. Thaler W, Watzka S, Martin F, et al. Preoperative staging of rectal cancer by endoluminal ultrasound vs. magnetic resonance imaging. Preliminary results of a prospective, comparative study. Dis Colon Rectum 1994;37:1189-93.

[14]. Onodera J, Maetani S, Nishikawa T, et al. The reappraisal of prognostic classifications for colorectal cancer. Dis Colon Recum 1989;32:609-14.

[15]. Ebner F, Kressel HY, Mintz MC, et al. Tumor recurrence versus fibrosis in the female pelvis: differentiation with MR imaging at 1.5T. Radiology 1988; 166:333-40.

[16]. De Lange EE, Fechner RE, Wanebo HJ. Suspected recurrent rectosigmoid carcinoma after abdominoperineal resection: MR imaging and histopathologic findings. Radiology 1989;170:323-8.

[17]. Limberg B. Diagnosis and staging of colonic tumors by conventional abdominal sonography as compared with hydrocolonic sonography. N Engl J Med 1992; 327:65-9. 